\section{APPLICATION OF NEW RULES FOR PATTERN SPECIFICATION ACCORDING ISO GPS \\ KAREL PETR}

Czech Technical University in Prague, Faculty of Mechanical Engineering, Department of Designing and Machine Components, Prague, Czech Republic

DOI : 10.17973/MMSJ.2020_11_2020010

karel.petr@fs.cvut.cz

This article is based on new rules used in ISO GPS standards (year 2017). All rules important for creating of tolerancing model (as dimension, roughness and geometrical specifications) was harmonized and more clearly defined across standards. Article provides rules for the tolerancing of a tolerance zone pattern, i.e. a collection of tolerance zones constrained to each other with or without reference to a datum system which doesn't lock all degrees of freedom, simultaneous requirement, combined zone etc. All rules shall respect the independency principle defined into ISO 8015. This article explains application of pattern specifications to specific components with detailed descriptions.

KEYWORDS

pattern specification, GPS, tolerance zone pattern, constraint, geometrical tolerancing, simultaneous requirement, drawing descriptions

\section{INTRODUCTION}

The ISO GPS (Geometrical Product Specifications) define an internationally uniform description language, that allows expressing unambiguously and completely all requirements for the micro and macro geometry of a product with the corresponding requirements for the inspection process in technical drawings, considering current possibilities of measurement and testing technology.

Engineering drawings without longitudinal tolerances, roughness, geometrical tolerances and datums or datum systems are in most cases incomplete, ambiguous, and therefore not unambiguously interpretable. The incomplete, ambiguous tolerancing of components in engineering drawings causes not only increased production and inspection costs but also makes impossible reasoned complaints of shortcomings and ultimately lead to an incalculable liability risk in the case of legal disputes. Therefore, the designers and metrology engineers must have good knowledge on ISO GPS and verification methodology which ought to be given during studies or refreshed and supplemented by specialized training courses on the new standards [Petr 2017].

ISO default specification operators and number of fundamental principles that apply to all GPS standards and technical product documentation are indicate in fundamental ISO GPS standards [ISO8015 2011].

Feature Principle and Independency Principle - Base principles according [ISO8015 2011] which are necessary to keep are:

Feature principle - a workpiece shall be considered as made up of a number of features limited by natural boundaries. By default, every ISO GPS specification for a feature or relation between features applies to the entire feature or features; and each ISO GPS specification applies only to one feature or one relation between features.

Independency principle - by default, every ISO GPS specification for a feature or relation between features shall be fulfilled independent of other specifications except when it is stated in a standard or by special indication (e.g. (M) in circle; $(L)$ in circle; (E) in circle; (R) in circle; CT; CZ; UF; ...) as part of the actual specification.

For the indication of specifications on the drawing is very important keep up rules (principles) description in standard [ISO8015 2011], especially feature principle and independency principle.

Fig. 1 shows basic standards about geometrical tolerancing (ISO 1101, ISO 2692, ISO 5459, ISO 5458, ISO 1660, ...), linear and angular tolerancing (ISO 286, ISO $14405-1$ to 3 , ISO 2768) and roughness (profile surface texture ISO 1302 or according new standard ISO 21920-1, areal surface texture ISO 25178-1).

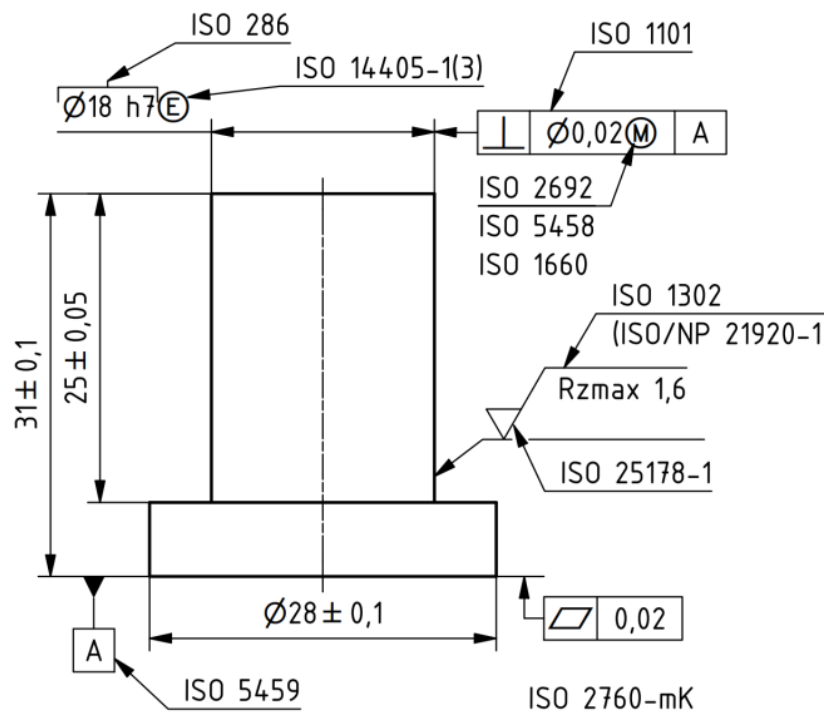

Figure 1. Geometrical specification according ISO standards with indication of number of standards used on drawing [Petr 2019]

\section{THEORY AND METHODS}

A geometrical specification applies to a single complete feature (see Fig. 2), unless an appropriate modifier is indicated. When the toleranced feature is not a single complete feature, see Fig. 2 (R↔S).

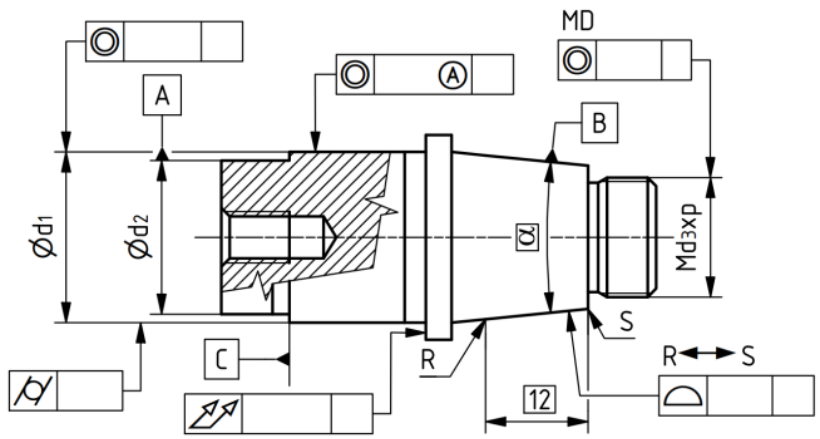

Figure 2. Geometrical specification (ISO1101:2017) applies to drawing [Petr 2017]

When the geometrical specification refers to the integral feature, the geometrical specification indication shall be connected to the toleranced feature by a reference line, and a leader line terminating according Fig. 2 (the bottom half of the figure). When the geometrical specification refers to a derived 
feature (a median point, a median line, or a median surface), it shall be indicated either by a reference line and a leader line terminated by an arrow on the extension of the dimension line of a feature of size or by modifier $(A)$ in circle, see Fig. 2 .

\subsection{New Specifications for 2D Drawing and 3D CAD Model according ISO 1101}

Main new specifications in ISO 1101 are (see Fig. 3): Intersection plane indicator; Orientation plane indicator; Direction feature indicator; Collection plane indicator [ISO1101 2017].

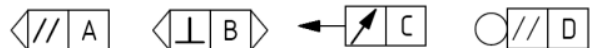

Figure 3. Intersection plane indicator (symbol with datum A); Orientation plane indicator (symbol with datum B); Direction feature indicator (symbol with datum C); Collection plane indicator (symbo with datum D)

With these specifications, disappeared dependence of placement of geometric tolerances on specific 2D view in orthographic projection (ISO E). The method of evaluation for metrology it was also better specified [Bronček 2017]. Used these specifications, we can better and more clearly positioned geometric tolerances on a 2D drawing or also into a parametric 3D CAD model using PMI dimensions.

More information about indicators see [Petr 2020] or [ISO1101 2017].

\subsection{Explanation of Toleranced Feature Specification Elements}

If the toleranced feature is not one complete single feature, this shall be indicated by using the tools given in standard [ISO1101 2017] [ISO1660 2017], e.g. the SZ, CZ, UF, "all over", "all around" and "between" specification elements. For example, symbols SZ and CZ are very important for applications in pattern specification.

Fig. 4 shows applications of symbols for tolerance zone (SZ Separate Zones, CZ - Combined Zone) and tolerance feature (UF - United Feature) [ISO1660 2017].

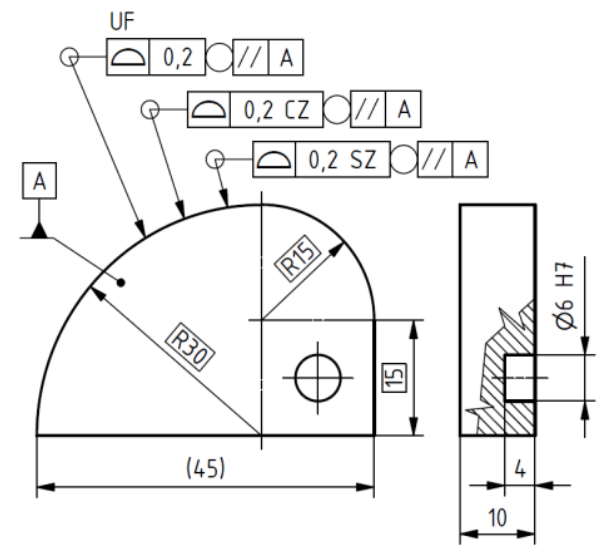

Figure 4. Example application of three types of symbols used for indication for tolerance feature, which isn't one complete single feature

\section{Surface Profile Specification for a Set of Independent Features}

The separate zones (SZ), modifier considers the set of single features as separate features (see Fig. 4), with unrelated tolerance zones. Since there are a number of toleranced features, there are an equal number of specified characteristics [ISO1660 2017].

Fig. 5 shows tolerance zones for separate features. The tolerance zones are each limited by two equidistant surfaces enveloping spheres $(s \varnothing 0.2)$ with a diameter equal to the tolerance value $T_{P P}(\varnothing 0.2$ - geometrical tolerance with modifier SZ in Fig. 4), the centres of which are situated on the TEF (theoretical exact feature). This results in one tolerance zone's limits being $90^{\circ}$ sections of coaxial cylinders with radius 14.9 and 15.1, respectively, one tolerance zones limits being $90^{\circ}$ sections of coaxial cylinders with radius 29.9 and 30.1, respectively, and two tolerance zones limits being planes separated by 0.2 .

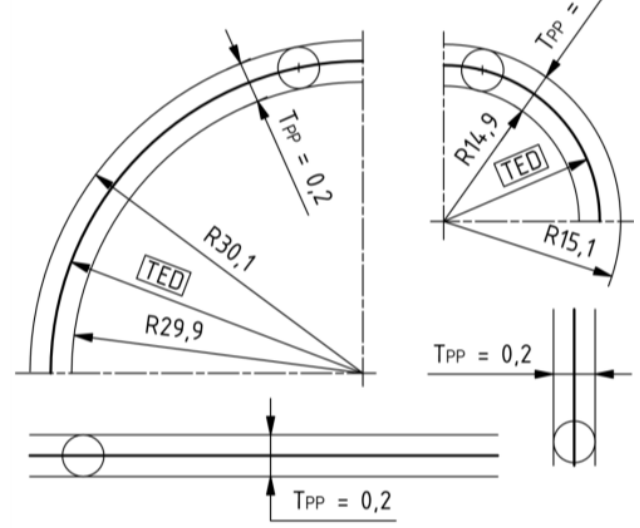

Figure 5. Tolerance zone for surface profile specification for a set of independent features (separate zones - SZ) according Fig. 4.

\section{Combined Surface Profile Specification for a Set of Features}

The combined zone (CZ), modifier considers the set of single features as separate features, but combines the tolerance zones (see Fig. 4). Since it builds a collection of toleranced features, it cannot define a derived feature, if the individual features do not have a derived feature. Therefore, the $C Z$ modifier is appropriate to use when the toleranced features function separately, but with a relation between them (see Fig. 6). The CZ modifier defines only one specified characteristic [ISO1660 2017].

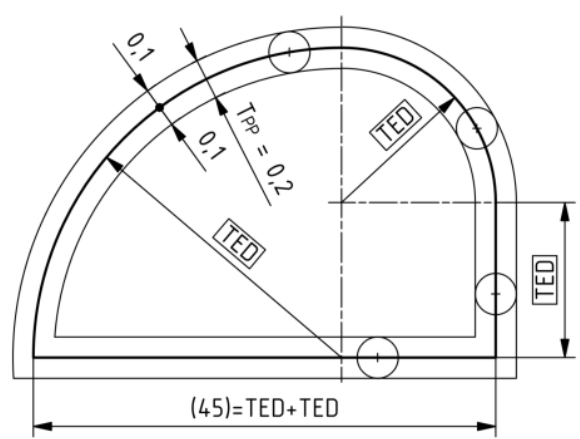

Figure 6. Effective combined tolerance zone for the set of single features (combined zone - CZ) according Fig. 4

Each tolerance zone is limited by two equidistant surfaces enveloping spheres $(s \varnothing 0.2)$ with a diameter equal to the tolerance value $T_{P P}(\varnothing 0.2$ - geometrical tolerance with modifier $\mathrm{CZ}$ in Fig. 4), the centres of which are situated on the TEF.

\section{Surface Profile Specification for a United Feature}

The united feature (UF), modifier builds one compound feature out of several single features (see Fig. 4). This compound feature may have a derived feature, even when the individual features do not. Therefore, the UF modifier is appropriate to use, when the function(s) is related to the integral compound feature considered as one feature, or to its derived feature. Modifier UF is not in tolerance indicator (zone and feature section), but in adjacent indications, because is targeted to feature [ISO1660 2017]. 


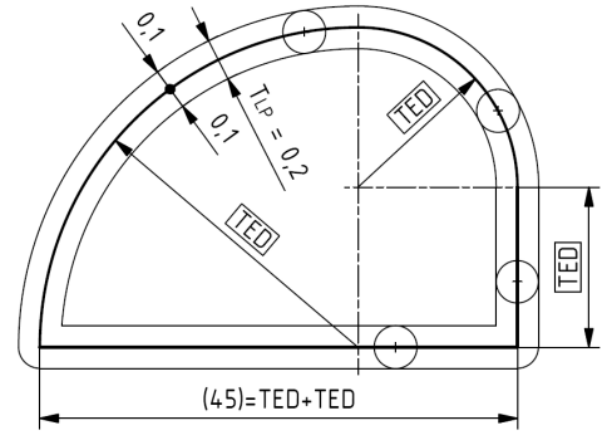

Figure 7. Tolerance zone for one compound feature (united feature UF) according Fig. 4

The tolerance zone (see Fig. 7) is limited by two equidistant surfaces enveloping spheres ( $\$ \varnothing 0.2)$ with a diameter equal to the tolerance value $T_{p p}(\varnothing 0.2$ - geometrical tolerance with modifier UF in Fig. 4), the centres of which are situated on the TEF. Because the periphery is considered one feature, the spheres that define the limits of the tolerance zone are rolled across the discontinuities in the feature and create round corners (see Fig. 7 - left and right corner below) in the tolerance zone on the outside of the discontinuities.

In the case of profile tolerancing of integral features, the practical difference between UF and CZ is small and limited to the shape of the tolerance zone in transitions between features. Conceptually, the UF, united feature, modifier builds one compound feature out of several features and creates one tolerance zone for that compound feature, whereas the $C Z$, combined zone, modifier considers the features separate, but combines the tolerance zones. The practical difference is small in this case, but may be significant in the case of patterns.

\subsection{Modifiers for Pattern Specification}

This part of article establishes selected rules for pattern specifications and for combine individual specifications (according [ISO5458 2018]), for geometrical specifications e.g. using the symbols Position, Symmetry, Line profile and Surface profile, as well as Straightness (only for derived feature - axis) and Flatness (only for derived feature - middle plane).

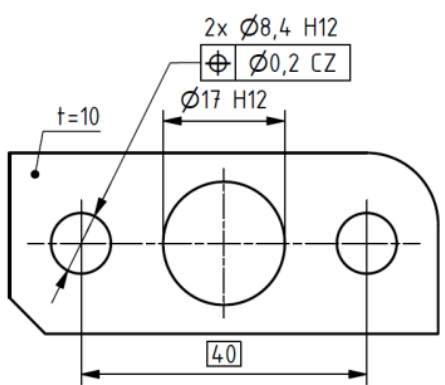

Figure 8. Example of pattern specification (because CZ modifier is used)

Pattern specification (see Fig. 8) combined requirement indicated by a set of geometrical specifications, and controlled by a tolerance zone pattern. The set of pattern features controlled by a pattern specification does not define a united feature (UF). For pattern specification are possible used Specification modifiers (symbols) indicated in Tab. 1. Modifier UF is possible applied to toleranced feature and CZ, SZ, SIMi, CZR is possible applied to tolerance zones [ISO5458 2018].

The tolerance zones defined by one tolerance indicator or by several tolerance indicators shall be considered independently by default (in terms of clarity, it is better to state modifier SZ).
In order to manage functional requirements for a set of features, they can be controlled simultaneously by means of a pattern specification, using tolerance zone pattern modifiers CZ, CZR, UF (features) or SIMi (Tab. 1) [ISO5458 2018]. There are two ways to create a tolerance zone pattern, either by using a single indicator pattern specification with the CZ or CZR modifiers or by using a multiple indicator pattern specification using SIM modifiers.

\begin{tabular}{|c|c|c|}
\hline Symbol & Description & Internal constraint \\
\hline SZ & Separate zones & None \\
\hline CZ & Combined zone & Orientation and location \\
\hline CZR & $\begin{array}{l}\text { Combined zone } \\
\text { rotational only }\end{array}$ & Only orientation \\
\hline SIMi & $\begin{array}{l}\text { Simultaneous } \\
\text { requirement No. i }\end{array}$ & Orientation and location \\
\hline UF & United feature & Not applicable \\
\hline
\end{tabular}

Table 1. Specification modifiers for pattern spec. [ISO5458 2018]

Fig. 8 shows position tolerance for two same holes and from this reason is defined a pattern specification (because $\mathrm{CZ}$ modifier is used).

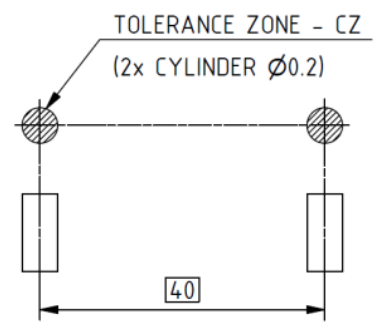

Figure 9. Tolerance zone for pattern specification according Fig. 8. Explanation of what indication on the drawing (view) means

The toleranced feature is the collection of two (because $2 x$ is indicated) extracted median lines, where each nominal median line is a straight line (axis of hole). The tolerance zone (see Fig. 9) is a tolerance zone pattern (CZ modifier) composed of two $(2 \mathrm{x})$ cylindrical zones with a diameter of $0.2 \mathrm{~mm}$, where their axes are constrained in orientation to be parallel (implicit TED / theoretically exact dimensions/ of $0^{\circ}$ ) and constrained in location to be $40 \mathrm{~mm}$ apart (explicit TED $=40 \mathrm{~mm}$ ), without external constraint coming from a datum (no datum or datum system).

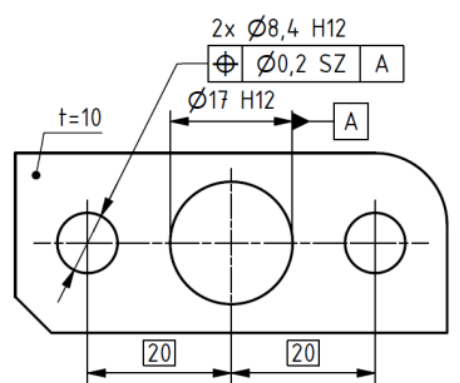

Figure 10. Example of the specification, which is not a pattern specification (because SZ modifier is used)

Fig. 10 shows position tolerance for two same holes with datum. The specification isn't a pattern specification (because SZ modifier is used). The toleranced feature is the collection of two $(2 \mathrm{x})$ extracted median lines, where each nominal median line is a straight line (axis of hole). Each individual tolerance zone is considered independently (SZ modifier) and does not constitute a tolerance zone pattern - see Fig. 11 (left view). 
Each tolerance zone is a cylindrical zone with a diameter of 0.2 $\mathrm{mm}$, where the axis is externally constrained in orientation to be parallel (implicit TED of $0^{\circ}$ ) and in location at a distance $20 \mathrm{~mm}$ ( $2 x$ explicit TED $=20 \mathrm{~mm}$ ) from the datum $A$ (axis of center hole) [ISO5459 2011]. The tolerance zones for the two toleranced features are independent and aren't constrained between them. The distance $40 \mathrm{~mm}(20 \mathrm{~mm}+20 \mathrm{~mm})$ isn't considered as an internal constraint between the tolerance zones (SZ modifier).
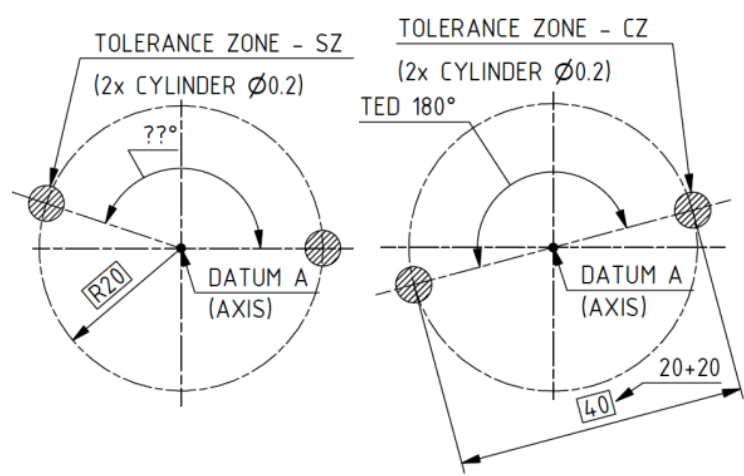

Figure 11. Tolerance zone for pattern specification according Fig. 10 view on left side and according Fig. 12 - view on right side. Explanation of what indication on the drawing (view) means

Fig. 12 shows position tolerance for two same holes with datum. The specification is a pattern specification (because $\mathrm{CZ}$ modifier is used). The toleranced feature is the collection of two $(2 \mathrm{x})$ extracted median lines, where each nominal median line is a straight line (axis of hole). The tolerance zone is a tolerance zone pattern ( $C Z$ modifier) composed of two (2x) cylindrical zones with diameters of $0.2 \mathrm{~mm}$, where their axes are internally constrained in orientation to be parallel (implicit TED of $0^{\circ}$ ) and in location at a distance $40 \mathrm{~mm}$ apart (2x explicit $\mathrm{TED}=20 \mathrm{~mm}$ and diametrically disposed) - see Fig. 11 (right view). Moreover, the tolerance zones are externally constrained in location from the datum A (axis of center hole) [ISO5459 2011] at a distance of TED $=20 \mathrm{~mm}$.

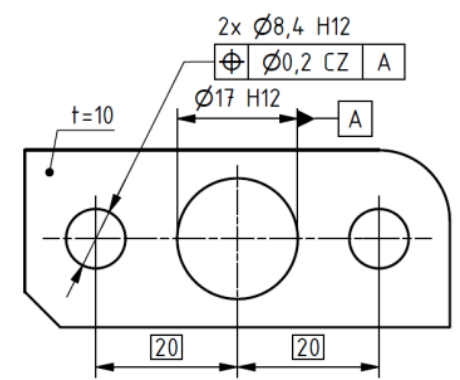

Figure 12. Example of pattern specification (because CZ modifier is used)

\subsection{Sequences of modifiers for Pattern Specification}

There shall be as many CZ/SZ/CZR indications as there are levels in the pattern (see Tab. 2) [ISO5458 2018]. Tab. 2 shows some examples of sequences of modifiers (SZ, CZ and CZR) and whether they have a meaning. CZ or CZR indications shall not precede an SZ indication. CZ indications shall not precede a CZR indication.

\begin{tabular}{|c|c|}
\hline Sequence & Meaning \\
\hline SZ SZ & Indicates independency of all features \\
\hline SZ CZ & $\begin{array}{l}\text { Indicates independency of tolerance zone } \\
\text { patterns (first level) }\end{array}$ \\
\hline CZ SZ & $\begin{array}{l}\text { Meaningless (in [Petr 2017] wrong } \\
\text { interpretation of this sequence is shown) }\end{array}$ \\
\hline
\end{tabular}

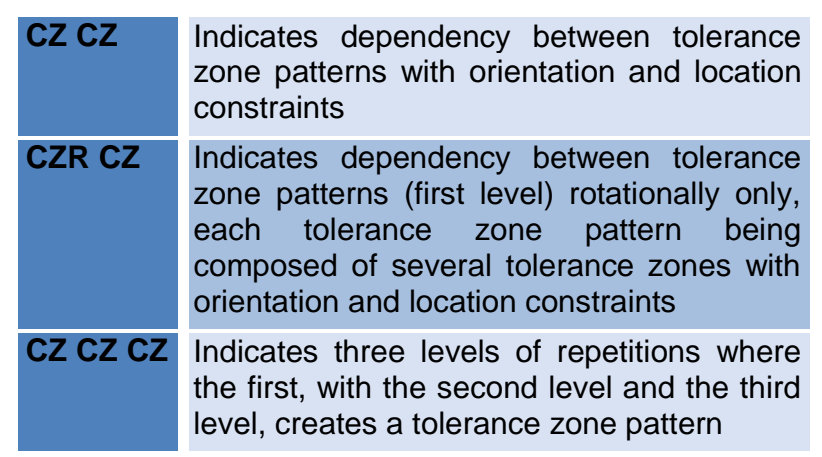

Table 2. Examples of sequences of modifiers and their meaning (selection of [ISO5458 2018])

Fig. 13 shown three variants of application sequences of modifiers with surface profile tolerance with "all-around" modifier shall be considered with a collection plane indicator [ISO1101 2017]. " $2 x$ " means number of identical groups (two squares profiles - prisms) and symbol "all around" means number of identical features (four identical surfaces) - see Fig. 13.

First variant of pattern specification [ISO5458 2018] - UF modifier and one CZ modifier (see Fig. 13). How was write in Chapter 2.2, when the modifier UF is placed above the tolerance indicator, the collection of the integral features is considered as a single feature (for both features $-2 \mathrm{x}$ ). It doesn't transform a single geometrical specification to a pattern specification. The toleranced feature is the collection of two united features (2x UF), each consisting of four extracted unified integral surfaces (all around symbol).

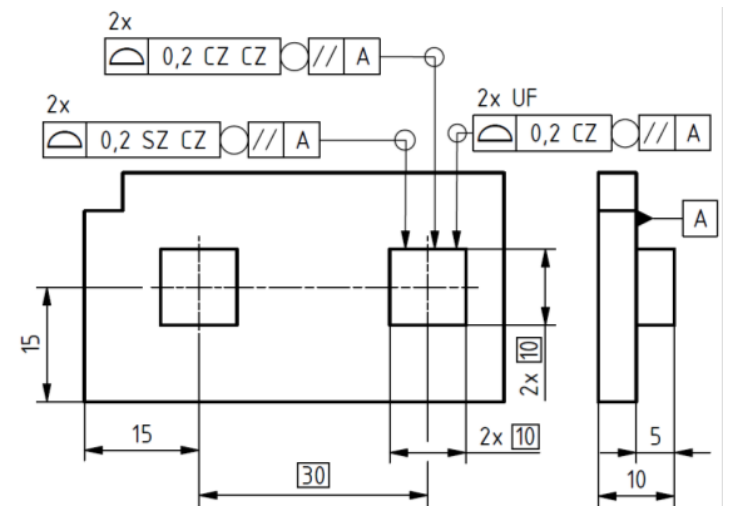

Figure 13. Example of pattern specification with sequences of modifiers (three variants)

Fig. 14 shows a tolerance zone pattern ( $C Z$ modifier) composed of two tolerances zones, each one consisting of two offset surfaces from the nominal shape of the united feature and constrained in orientation to be parallel (implicit TED of $0^{\circ}$ ) and in location to be TED $=30 \mathrm{~mm}$ apart in one direction (explicit TED $=30 \mathrm{~mm}$ ) and $0 \mathrm{~mm}$ in another perpendicular direction (implicit TED) without external constraint coming from a datum (datum system).

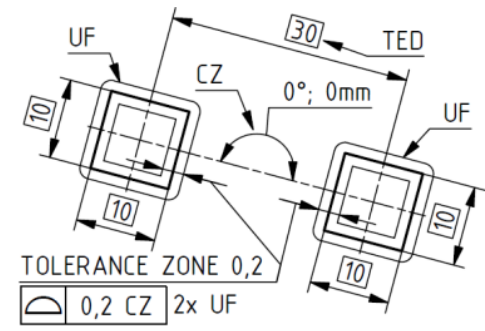

Figure 14. Tolerance zone for pattern specification according Fig. 13 2x UF and one CZ modifier 
Second variant of pattern specification [ISO5458 2018] sequences of modifiers CZ CZ (see Fig. 13). Fig. 15 shows two (2x) pattern specifications (last CZ in sequences $C Z C Z$ ) which are dependent (first $C Z$ in sequences $C Z C Z$ ), creating a global pattern specification. The toleranced feature is the collection of eight extracted integral surfaces [Petr 2017].

Fig. 15 shows a tolerance zone pattern consisting of two tolerance zone patterns ( $C Z \mathrm{CZ}$ ), composed of four tolerance zones, space between two parallel planes $0.2 \mathrm{~mm}$ apart and constrained in orientation (implicit TEDs $4 \times 90^{\circ}$ ) and in location 2x $10 \mathrm{~mm}$ apart (explicit TEDs). The two tolerance zone patterns are constrained in orientation to be parallel (implicit TED of $0^{\circ}$ ) and in location to be TED $=30 \mathrm{~mm}$ apart in one direction (explicit TED $=30 \mathrm{~mm}$ ) and aligned (implicit TED of 0 $\mathrm{mm}$ apart) in the perpendicular direction, without datum (external constraint)

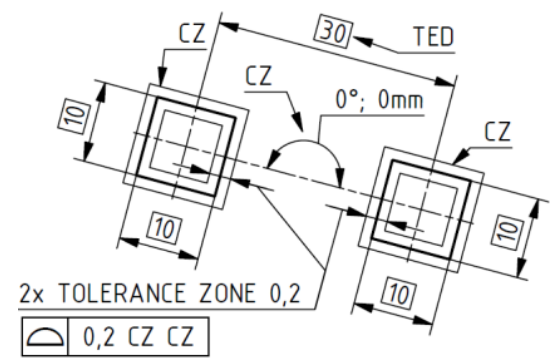

Figure 15. Tolerance zone for pattern specification according Fig. 13 sequences of modifiers CZ CZ

Third variant of pattern specification [ISO5458 2018] sequences of modifiers SZ CZ (see Fig. 13). Fig. 16 shows two ( $2 x$ or two leader lines) pattern specifications ( $C Z$ in the sequence SZ CZ) considered independently (first SZ in the sequence). For each pattern specification, the toleranced feature is the collection of four extracted integral surfaces (all around symbol) [Petr 2017]. Sequence of modifiers CZ SZ is meaningless.

Fig. 16 shows the tolerance zone pattern is composed of four tolerances zones, constrained between them in orientation (implicit TED $0^{\circ}$ and $90^{\circ}$ ) and in location to be $10 \mathrm{~mm}$ (in one direction) and $10 \mathrm{~mm}$ (in another perpendicular direction) apart, with explicit TEDs, without datum.

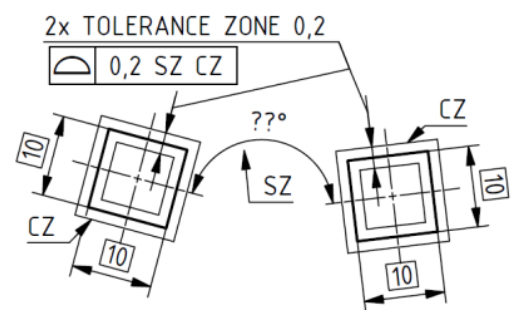

Figure 16. Tolerance zone for pattern specification according Fig. $13-$ sequences of modifiers SZ CZ (in this case TED $30 \mathrm{~mm}$ isn't TED)

\subsection{Simultaneous requirement - SIMi}

The set of geometrical specifications, linked together with the indication of SIMi (not independent), defines a pattern specification (SIM1 modifier) [ISO5458 2018] - see Fig. 17.

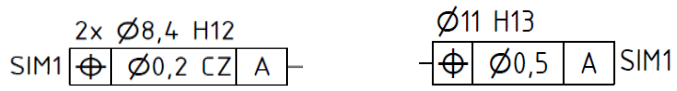

Figure 17. Example of indication of SIMi form left or right side of tolerance indicator

Fig. 18. shows example of desk workpiece without SIMi, but when jointed Fig. 17 and Fig. 18 - example with SIMi. The SIMi modifier adjacent to the two tolerance indicators means that the two tolerance zone patterns are combined into a single requirement. All three tolerance zones are locked together by location and orientation constraints.

The tolerance indicator with $\mathrm{CZ}$ defines (Fig. 18), as toleranced feature, a set of two extracted median lines, and, as tolerance zone, a tolerance zone pattern defined by two cylindrical zone - same application as is shows in example in Fig. 12. The tolerance indicator without $\mathrm{CZ}$ defines as toleranced feature an extracted median line and as tolerance zone a cylindrical zone.

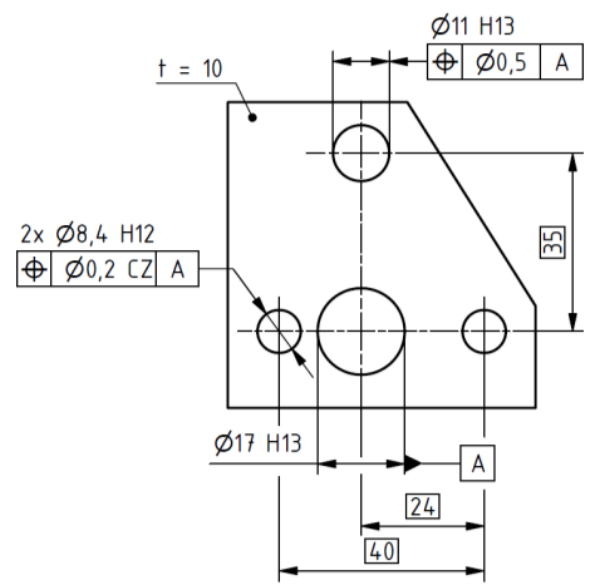

Figure 18. Example of indication of SIMi on desk workpiece

Without SIMi modifier (SIM1 in this case), the two specifications are independent: the datum A doesn't lock all degrees of freedom of the tolerance zone pattern and the cylindrical zone of diameter $0.5 \mathrm{~mm}$ can be moved rotationally relatively ( $\alpha \neq 90^{\circ}$ see Fig. 19). We can say that both geometric tolerances (Fig. 18) are independent of each other, there does not have to be an angle of 90 between them.

With SIMi modifier, the cylindrical zone of diameter $0.5 \mathrm{~mm}$ and the tolerance zone pattern aren't independent. They are constrained in orientation (implicit TED of $0^{\circ}$ ) and in location (explicit TED of $35 \mathrm{~mm}$ and implicit TED of $90^{\circ}$ ), combining these specifications in one pattern specification (see Fig. 19). We can say that both geometric tolerances (Fig. 18 with application Fig. 17 - SIM1) are interdependent, there must be an angle of 90 between them.

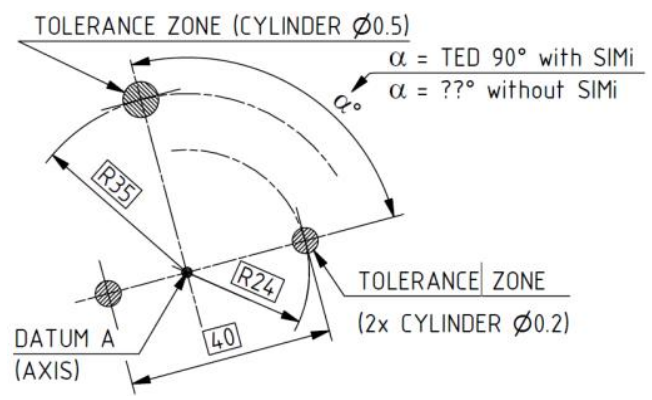

Figure 19. Tolerance zone for pattern specification according Fig. 18 with/without SIM1 indication

\section{DISCUSSION - EXAMPLES OF PREVIOUS PRACTICE AND NEW/CURRENT PRACTICE}

By default, based on the independency principle defined in ISO 8015, a geometrical specification of form, orientation, location or run-out, without modifier, applied to " $\mathrm{n}$ " geometrical features (" $n$ " being greater than one) is equivalent to " $n$ " independent geometrical specifications: each geometrical feature shall be considered individually and each specification shall be considered individually (independent between them). The resulting independent tolerance zones 
correspond to an implicit indication of the separate zone (SZ) modifier: the "all around" modifier does not create itself a united feature or a pattern.

Positional tolerance for several geometrical features (Fig. 20).

When positional tolerancing is applied to several geometrical features and all the non-redundant degrees of freedoms of the tolerance zones are unlocked (Fig. 20 - position tolerance with $\mathrm{CZ}$ ), either the separate zone (SZ) modifier or the combined zone (CZ) modifier shall always be indicated in the second compartment of the tolerance indicator. To create one homogeneous pattern, the $\mathrm{CZ}$ modifier shall be indicated in the tolerance section of the tolerance indicator - see Fig. 20 (position tolerance with CZ). The previous practice was without application of $\mathrm{CZ}$ modifier and the idea was the same as when using the $C Z$ modifier. To create a new level of pattern defined as a homogeneous pattern of more than one homogeneous pattern, an additional CZ modifier shall be indicated after the sequence defining the previous level of pattern. One $C Z$ in the tolerance section defines a single pattern. The sequence $C Z$ in the tolerance in the tolerance section defines a pattern of patterns (pattern of Level 3 - see Fig. 13).
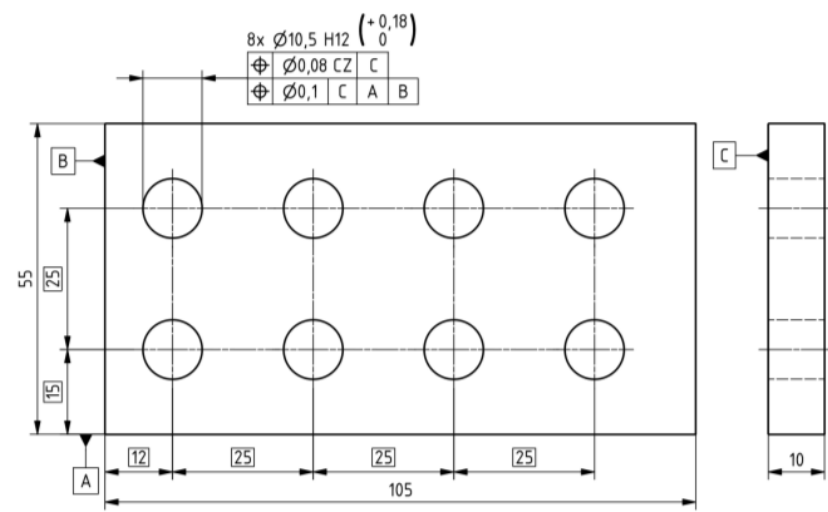

Figure 20. Positional tolerance is applied for several geometrical features

The former practices used in examples of chapter 2.3.

The former practice for Fig. 8 (resp. Fig. 12) was without the CZ modifier. The tolerance zones defined by one tolerance indicator or by several tolerance indicators without CZ (isn't a pattern - see Fig. 10) shall be considered independently by default (in terms of clarity, it is better to state modifier SZ - rule from ISO 5458) - comparison of the resulting tolerance zones with $\mathrm{CZ}$ and SZ see Fig. 11.

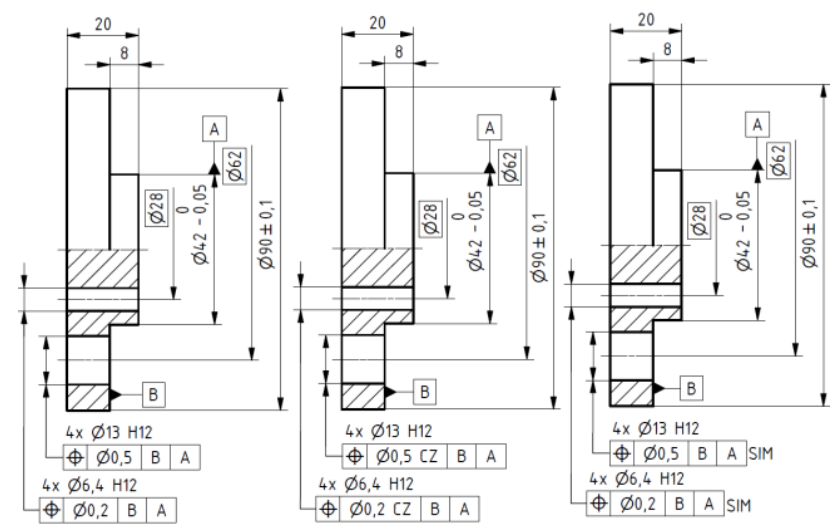

Figure 21. Indication for position specifications for two groups of features circularly disposed, with the same axis and without simultaneous requirement (middle view). Indication for position specifications for two groups of features circularly disposed, and with the same axis and with simultaneous requirement (right view). The previous practice was without application of CZ modifier or SIM (left view.)
Positional tolerance for two groups of several geometrical features (Fig. 21).

It was former practice to consider a set of features equally arranged around an axis (i.e. in a complete circle) and toleranced with one or several position specifications controlled by a unique tolerance zone pattern when they were not all related to any datum or when they were all related to the same datum system, whatever the tolerance values (see Fig. 21 left view). This former practice has been replaced by the use of CZ modifier (see Fig. 21 middle view) when all position specifications have the same tolerance value or SIMi (SIM in this case) modifier (see Fig. 21 right view) when at least one position specification has a tolerance value different from the others.

The SIM modifier should be used in order to unambiguously identify which groups of tolerance zone patterns shall be regarded simultaneously as a single tolerance zone pattern (Fig. 21 right view).

Special statements such as "angular location optional" are no longer necessary to indicate that there are several independent pattern specifications.

\section{Positional tolerance for eight grooves (Fig. 22).}

In this example (see Fig. 22), the meaning does not change if the characteristic symbol is the position symbol or the symmetry symbol (the symmetry symbol does not need to show the drawing line to see the implicit TEDs of $0 \mathrm{~mm}$ from datum $A$, as requested with position symbol).

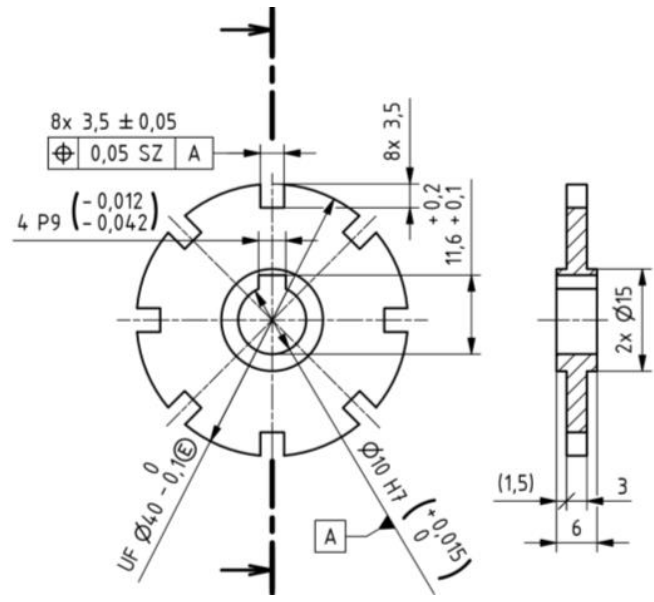

Figure 22. Positional tolerance for eight grooves with SZ or CZ

The toleranced feature is the collection of the eight extracted derived surfaces identified by the upper indication above the tolerance indicator $(8 \times 3,5 \pm 0,05)$. For each of the eight $(8 x)$ toleranced features considered independently (SZ, disregarding the angles between them), the extracted median surface shall be contained between two parallel planes 0.05 apart, which are symmetrically disposed about the theoretically exact position of the median plane, with respect to datum axis A. With the $S Z$ specification element, the angles between the tolerance zones for the eight slots are not locked. If the $\mathrm{CZ}$ specification element had been used instead, the tolerance zones would have been locked at $45^{\circ}$ intervals (the implicit angle of $45^{\circ}=$ $\left.360^{\circ} / 8\right)$. The previous practice was without application of $\mathrm{CZ}$ or SZ modifier and the idea was the same as when using the $\mathrm{CZ}$ modifier.

Position tolerance for flanged holes (Fig. 23).

The toleranced feature is the collection of four $(4 \mathrm{x})$ extracted median lines. The tolerance zone is a tolerance zone pattern (CZ modifier) composed of four cylindrical zones with diameters of $1 \mathrm{~mm}$, where their axes are internally ( $C Z$ modifier) 
constrained in orientation (implicit TED angle of $90^{\circ}=360^{\circ} / 4$ ) and in location at a distance $70 \mathrm{~mm}$ apart (explicit TED diameter $70 \mathrm{~mm}$. The previous practice was without application of $C Z$ modifier and the idea was the same as when using the $C Z$ modifier.

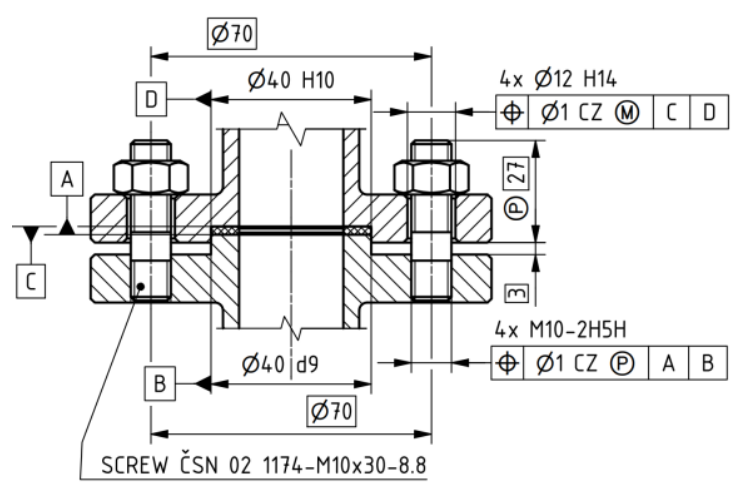

Figure 23. Positional tolerance for flanged holes (CZ modifier needed)

\section{The difference between CZ and CZR (Fig. 24).}

Fig. 24 provides examples which illustrate the internal constraints introduced by the CZ or CZR modifiers and the external constraints introduced by the datum or datum system. For both variants the toleranced feature is the collection of four (4x) extracted integral surfaces. For the first specification with CZR modifier - the tolerance zone pattern is composed of four single tolerance zones (two parallel planes 0.1 apart) constrained between them in orientation only (CZR) to be perpendicular and parallel (implicit TED $0^{\circ}$ and $90^{\circ}$ ). For the second specification with $\mathrm{CZ}$ modifier - the tolerance zone pattern is composed of four single tolerance zones (two parallel planes 0.2 apart) constrained between them (CZ) in orientation $\left(0^{\circ}\right.$ and $\left.90^{\circ}\right)$ and in location $(32 \mathrm{~mm}$ and $32 \mathrm{~mm})$. The previous practice was without application of $\mathrm{CZ}$ modifier (the idea was the same as when using the CZ modifier) and without CZR modifier and apply tolerance on each feature separately.
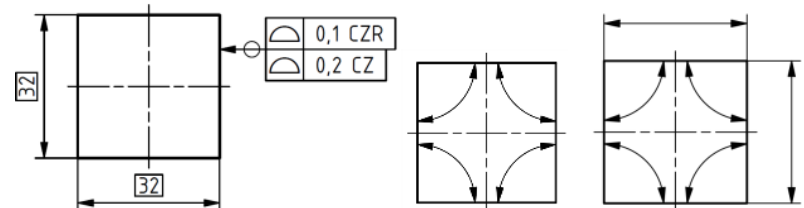

Figure 24. The difference between CZ and CZR. Indication on drawing (left view), internal constraints in the tolerance zone pattern with CZR (middle view) and CZ (right view)

Indication of multi-level single indicator pattern specification (Fig. 25)

Fig. 25 shows four specifications in VARIANT-1 (VARIANT-2 is alternative indication with the same meaning). The specifications of VARIANT-1 from top to bottom are described below.

The first specification (SZ CZ with datum A - orientation) controls four independent pattern specifications $(4 \mathrm{x} K)$. For each pattern specification, the first specification is defined as toleranced feature, the collection of four extracted median lines; as tolerance zone, the tolerance zone pattern (combined zone) composed of four cylindrical zones $(4 \mathrm{x} \mathrm{J})$ of diameter 0,1 $\mathrm{mm}$ with orientation constraint (parallel between them) and with location constraint between them at $12 \mathrm{~mm}$ apart.

The second specification ( $C Z C Z$ with datum $A$ - orientation) controls four dependent pattern specifications $(4 \mathrm{x} K)$ resulting in only one pattern specification considering: as toleranced feature, the collection of sixteen extracted median lines; as tolerance zone, the tolerance zone pattern (combined zone) composed of sixteen cylindrical zones $(4 x 4 x J)$ of diameter 0,3 $\mathrm{mm}$ with orientation constraint (parallel between them) and with location constraint between according drawing (vertical direction $12 \mathrm{~mm}$ and $16 \mathrm{~mm}$; horizontal direction $12 \mathrm{~mm}$ and $16 \mathrm{~mm})$.

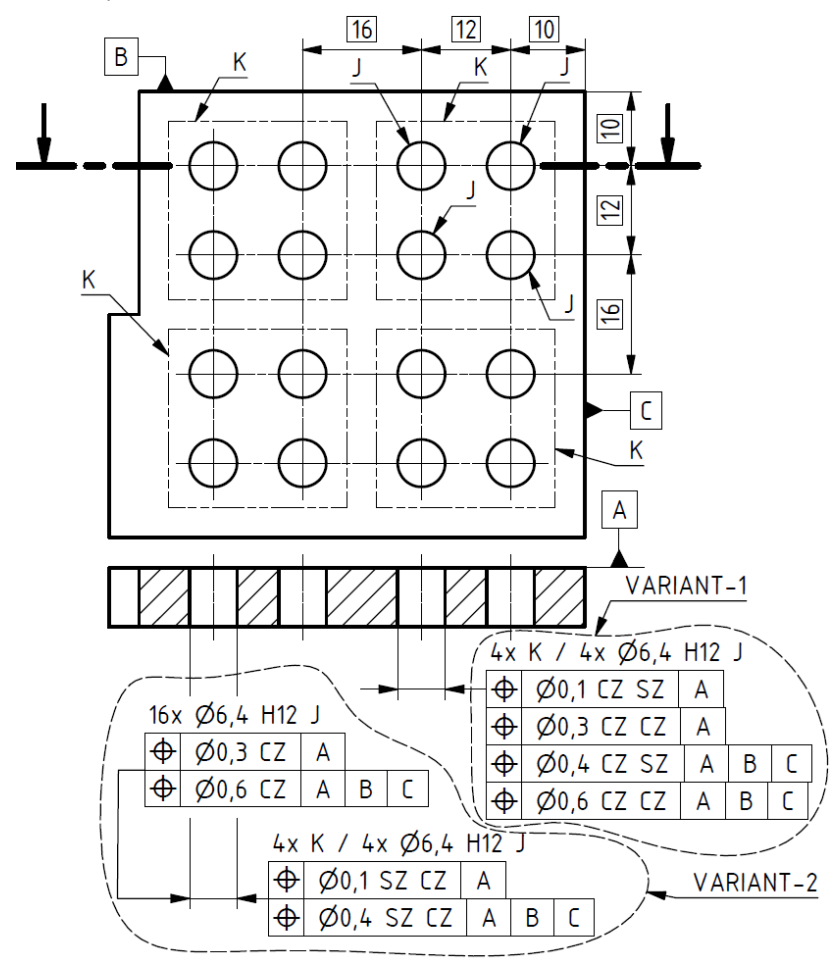

Figure 25. Indication of multi-level single indicator pattern specification (first level $(4 \mathrm{x} \mathrm{J})$ - number of identical features; second level $(4 \mathrm{x} \mathrm{K})$ number of identical groups)

The third specification (CZ SZ with datum system A, B, C) defines four $(4 \mathrm{x} \quad \mathrm{K})$ independent pattern specifications constrained in location from datums $B$ and $C$ (orientation A). For each pattern specification, the third specification is defined as toleranced feature, the collection of four extracted median lines; as tolerance zone, the tolerance zone pattern (combined zone) composed of four cylindrical zones ( $4 \mathrm{xJ}$ ) of diameter 0,3 $\mathrm{mm}$ with orientation constraint (parallel between them) and with location constraint between them $12 \mathrm{~mm}$ apart and constrained from datum $B$ and $C$.

The fourth specification ( $C Z C Z$ with datum system $A, B, C$ ) controls four $(4 x \quad K)$ dependent pattern specifications, constrained in location from datum $B$ and $C$ (orientation A), resulting in only one pattern specification considering as toleranced feature, the collection of sixteen extracted median lines; as tolerance zone, the tolerance zone pattern (combined zone) composed of sixteen cylindrical zones of diameter 0,6 $\mathrm{mm}$ with orientation constraint (parallel between them) and with location constraint between according drawing.

Simultaneous requirement for four surfaces (Fig. 26).

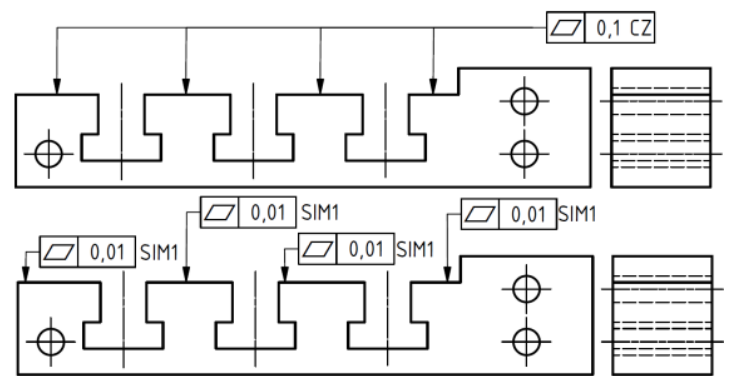

Figure 26. Simultaneous requirement for four surfaces. Above - single indicator pattern specification; below - multiple indicator pattern specification 
The use of the concept of "simultaneous requirement" transforms a set of more than one geometrical specification into a combined specification, i.e. a pattern specification. There are two ways to create a tolerance zone pattern, either by using a single indicator pattern specification with the CZ or CZR modifiers (former practise) or by using a multiple indicator pattern specification using SIM modifiers (new practise) - see Fig. 26.

\section{Simultaneous requirement for several features (Fig. 27)}

If the four pattern specifications on Fig. 27 were indicated without any SIM modifiers, the four tolerance zone patterns would be mutually unconstrained. Each of the four pattern specifications shall be considered independently of the others. All four tolerance zone patterns are constrained externally to datum $A-A$, but are rotationally independent from each other, i.e. without consideration of implicit angular TEDs between the four tolerance zone patterns.

Fig. 27 shows there are two simultaneous requirements defined by the indication of SIM1 and SIM2. Each simultaneous requirement shall be considered individually.

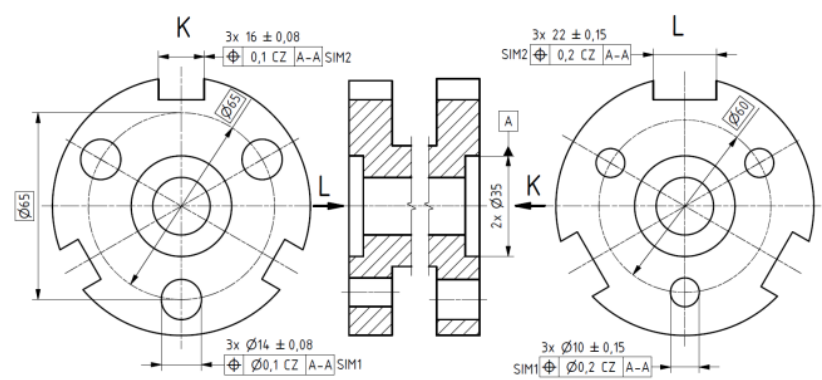

Figure 27. Simultaneous requirement for several features.

SIM1: the two specifications linked together with the SIM1 indication each use a $\mathrm{CZ}$ modifier to create a tolerance zone pattern. One of them is a pattern of three $\varnothing 0,1$ tolerance zones for the three extracted median lines of the $\varnothing 14$ holes, and the other is a tolerance zone pattern of three $\varnothing 0,2$ tolerance zones for the three extracted median lines of the $\varnothing 10$ holes. The SIM1 modifier locks the two tolerance zone patterns together into a combined tolerance zone pattern of six $(3 x+3 x)$ cylindrical tolerance zones. All six tolerance zones are constrained with the following internal constraint and external constraints (new practise, former practise not exist).

The SIM2 modifier locks the two tolerance zone patterns together into a combined tolerance zone pattern of six $(3 x+3 x)$ tolerance zones. All six tolerance zones are constrained with the following internal constraints and external constraints (new practise, former practise not exist).

\section{CONCLUSIONS}

Former practice covered geometrical specification with position characteristic symbol only and was based on an exception from the independency principle. For example, a position specification for a group of " $n$ " geometrical features, that was unrelated to a datum or related to a datum or a datum system that did not lock all degrees of freedom and with no modifier indicated in the tolerance section, defined an implicit tolerance zone pattern (the term "pattern" was not yet defined). Former practice appeared to avoid ambiguities, but it was not true, because sometimes there were ambiguities between designer and metrologist or sometimes also between designers alone.

Pattern specification used in this article and in the standard ISO 5458 apply to geometrical specifications not only with position symbol characteristics but also with line profile, surface profile, straight-ness, flatness and symmetry characteristic symbols. However, to create a pattern specification, a CZ modifier can be indicated in the second compartment of the tolerance indicator (the designer must respect the independency principle and feature principle).

To avoid ambiguity on a geometrical specification (new practise), using a position characteristic symbol applied to more than one geometrical feature, either a SZ or a CZ or a CZR modifier should be indicated in the tolerance section. The application of one of these modifiers is very important if a geometric specification is used for several features and at least one non-redundant degree of freedom of the tolerance zone is unlocked by the datum system. Thus, the CZ modifier must be used in cases of patterns that do not have all degrees of freedom taken away (e.g. in the form of a base system) - new practise. The tolerance zones defined by one tolerance indicator or by several tolerance indicators shall be considered independently by default; this corresponds to the meaning of the modifier SZ (new practise).

Special statements such as "angular location optional" are no longer necessary to indicate that there are several independent pattern specifications (SZ modifier).

The use of the concept of "simultaneous requirement" transforms a set of more than one geometrical specification into a combined specification, i.e. a pattern specification. So, the SIMi modifier should be used in order to unambiguously identify which groups of tolerance zone patterns (different groups) shall be regarded simultaneously as a single tolerance zone pattern. In contrast, the CZ modifier can be used only for the same features.

So, based on this article and on the standard ISO 5458, there are two ways to create a tolerance zone pattern, either by using a single indicator pattern specification with the CZ or CZR modifiers or by using a multiple indicator pattern specification using SIMi modifiers.

If older drawings are being modified for new production (new drawing creation date, it is necessary to add the CZ modifier to the geometrical tolerance indicator (zone, feature and characteristic section) when tolerating the pattern specification. Furthermore, it is possible to evaluate the possibility of using SIMi to obtain better parameters of the final component. A great advantage of the options presented in this article is the clearer application of geometric requirements (specifications) to the workpiece. Thanks to the above possibilities, ambiguities between the designer and the metrologist can be eliminated. Sometimes these ambiguities can be eliminated between the designers themselves.

\section{REFERENCES}

[Broncek 2017] Broncek, J., et al. The Application of New Rules of GPS in Structural Product Requirement. In: Proceedings of 58th International Conference of Machine Design Departments - ICMD 2017. 58th International Conference of Machine Design Departments. Praha, Horoměřice, 06.09.2017 - 08.09.2017. Prague: Technical Faculty. 2017, pp. 284-289. ISBN 978-80-2132769-6. Available from: http://2017.icmd.cz/proceedings/10_ICMD.pdf

[ISO8015 2011] Standard ISO 8015:2011 Geometrical product specifications (GPS) - Fundamentals - Concepts, principles and rules.

[ISO5459 2011] Standard ISO 5458:2018. Geometrical product specifications (GPS) - Geometrical tolerancing - Datums and datum systems 
[ISO1101 2017] Standard ISO 1101:2017. Geometrical product specifications (GPS) - Geometrical tolerancing - Tolerances of form, orientation, location and run-out.

[ISO1660 2017] Standard ISO 1660:2017. Geometrical product specifications (GPS) - Geometrical tolerancing - Profile tolerancing.

[ISO5458 2018] Standard ISO 5458:2018. Geometrical product specifications (GPS) - Geometrical tolerancing - Pattern and combined geometrical specification.

[Petr 2017] Petr, K. Indication of Functional Dimension According ISO GPS - How Shall We Applicate? In: Proceedings of 58th International Conference of Machine Design Departments - ICMD 2017. 58th International Conference of Machine Design Departments, Praha, Horoměřice, 2017-0906/2017-09-08. Prague: Technical Faculty. 2017, pp 284-289.

\section{CONTACTS:}

Ing. Karel Petr, Ph.D.

Czech Technical University in Prague

Faculty of Mechanical Engineering

Department of Designing and Machine Components

Technicka 4, 16607 Prague 6, Czech Republic

e-mail: karel.petr@fs.cvut.cz

Telephone: +420 224352415

http://konstruovani.fs.cvut.cz/
ISBN 978-80-213-2769-6. Available from: http://2017.icmd.cz/proceedings/56_ICMD.pdf.

[Petr 2019] Petr, K. Geometrical Tolerances According to ISO GPS. Praha: Verlag Dashöfer, 2019. ISBN 978-80-7635-019-9.

[Petr 2020] Petr, K. Explanation of New Specifications Used in Geometric Dimensioning Model. In: Current Methods of Construction Design, Proceedings of the ICMD 2018. THE 59TH INTERNATIONAL CONFERENCE OF MACHINE DESIGN DEPARTMENTS, Wellness Hotel Chopok, Demänovská dolina, 2018-09-11/2018-09-14. Cham: Springer International Publishing, 2020. p. 139-147. ISSN 2195-4356. ISBN 978-3-03033145-0. DOI 10.1007/978-3-030-33146-7_17. 